\title{
Label-free detection of DNA molecules moving inmicro-fluidic channels by infrared absorption spectroscopy
}

 \\ Hirano-Iwata ${ }^{\mathrm{c}, \mathrm{d}}$,Yasuo Kimura ${ }^{\mathrm{d}, \mathrm{e}}$, and Michio Niwano ${ }^{\mathrm{a}, *}$
}

${ }^{a}$ Laboratory for Nanoelectronics and Spintronics, Research Institute of Electrical Communication, Tohoku University, 2-2-1 Katahira, Aoba-ku, Sendai 980-8577, Japan

${ }^{b}$ Graduate School of Engineering, Tohoku University, 6-6 Aoba, Aramaki, Aoba-ku, Sendai 980-8579, Japan

${ }^{c}$ Graduate School of Biomedical Engineering, Tohoku University, 6-6 Aoba, Aramaki, Aoba-ku, Sendai 980-8579, Japan

${ }^{d}$ JST-CREST, 4-1-8 Honcho, Kawaguchi, Saitama 332-0012, Japan

${ }^{e}$ Faculty of Engineering, Tokyo University of Technology, 1404-1 Katakura, Hachioji, Tokyo 192-0914, Japan

\footnotetext{
${ }^{\dagger}$ Hitachi Cooporation, Japan

${ }^{\ddagger}$ Hang-Ichi Cooporation, 1-7 Honcho, Naka-ku, Yokohama 231-0005, Japan

*Corresponding author: Phone:+81-22-217-5501, FAX:+81-22-217-5501,

E-mail: niwano@riec.tohoku.ac.jp
} 


\begin{abstract}
Infrared absorption spectroscopy (IRAS) in amultiple internal reflection (MIR) geometry is integratedwith silicon-based micro-fluidic channelsto allow detection and separation of DNA molecules in the micro-channels. The micro-channels were fabricated on a Si MIR-prism using the conventional silicon lithographic techniques. In the present method we can probe solution-phase biomolecular species through IR spectral profiles of those biomolecules, allowing us to detect and separate biomolecules without fluorescence labeling.The applicability of this approach isdemonstrated with a representative test case: transport of single-stranded DNA molecules (oligonucleotides) in the micro-fluidic channels.
\end{abstract}

Keywords: Micro-fluidic channel, DNA, Infrared spectroscopy 


\section{Introduction}

Development of novel techniques of separating biological molecules is required for 'high-speed' and 'high-resolution' analysis of genetic information [1-5]. Micro-structures and channels fabricated by the use of the matured semiconductor nanotechnology are widely used in separation techniques such as 'micro-chip-electrophoresis' [6-8]. In the conventional method, biomolecules are separated in micro-channels by their size and quantity of their electric charges, and the separation is monitored via fluorescence signals from tags attached to the molecules $[9,10]$. However, fluorescent tags might have undesirable influences on biomolecular interactions, and moreover, labeling of molecules with fluorescent tags is time-consuming. To overcome these problems, we propose a novellabel-free detection scheme using infrared absorption spectroscopy (IRAS). In this scheme, we can detect and distinguish biomolecules through infrared absorption spectral profiles of the biomolecules, instead of fluorescent signals from labeled molecules. IRAS is in manyways an ideal detection technique, since virtually all molecules ofinterest absorb IR radiation. In contrast to UV/vis absorption, IRAS is a fingerprinting method. Characteristic functional groups areapparent by quick inspection of IRAS spectral profiles, and the uniqueIR signature of many molecules allows identification of munknown chemical speciesvia comparison with spectral libraries [11-13].Previously, we have shown thatDNA molecules can be in-situdetected in aqueous solution using IRAS in the multiple internal reflection geometry (MIR-IRAS) [14-16]. In the MIR method [17], a focused infrared light beam goes through a Si prism, internally reflecting many times, and biomolecular species in aqueous solution can be monitored by measuring IRAS spectra of the biomolecules in the vicinity of the prism surface where the evanescent field is present.

In this study, we apply MIR-IRAS to the detection of DNA molecules during electro-osmosis or electrophoresis in micro-fluidic channels. In Fig.1 is schematically 
shownthe principle of our detection scheme. Micro-fluidic channels are formed on a Si prism (crystal) through which a focused infrared light beam penetrates, internally reflecting many times. When the infrared light is totally reflected at the surface, the evanescent field is generated in the vicinity of the prism surface. A solution containing DNA molecules flowsthrough the micro-fluidic channels. When the molecules cross the evanescent field of infrared light, they produce infrared absorption signals which are fingerprints of the molecules of interest.One can therefore detect and distinguish DNA molecules by in-situ collecting infrared absorption spectra. The advantage of our method is thatthe MIR-IRAS method is very surface sensitive. The evanescent field is confined within sub-microns from the surface, and the exponential decayof the evanescent IR wave causes surface-associated species tobe weighted more heavily in sampling than their solution-phasecounterparts.

In the present study, we fabricate micro-fluidic channels on a Si prism surface using the conventional lithographic method, and demonstrate thattransport of single-stranded DNA in the micro-channels can be monitored through infrared spectral profiles.

\section{Experiment}

\subsection{Fabrication of micro-fluidic channels}

Our micro-fluidic channelsconsist of a Si substrate (Si MIR-prism) with sizes of $35 \times 20 \times 0.5 \mathrm{~mm}^{3}$ and a quartz substrate with $0.13 \mathrm{~mm}$ in thickness. We formed micro-fluidic channels on the Si substrate. The procedure thatwe employed for the formation of micro-fluidic channels (micro-capillaries) is schematically shown on the left side ofFig. 2. First, we prepared a Si MIR prism in which a focused infrared light beam penetrates, internally reflecting many times. The prism has two beveled edges through which an infrared light beam enters and exits the prism. Second, we formed a silicon dioxide layer $\left(\mathrm{SiO}_{2}\right)$ with a thickness of approximately $1.5-\mu \mathrm{m}$ on the prism surface. After that, we used the conventional 
lithographic method to form micro-fluidic channels on the $\mathrm{SiO}_{2}$ layer. Each channel was 1.5 $\mu \mathrm{m}$ in height and $200 \mu \mathrm{m}$ in width. The Si prism surface in the micro-fluidic channels may contact with chemically-active solution and should be inert against chemical attack by the $\underline{\text { solution. We therefore formed a passivation layer of } \mathrm{SiO}_{2}} \underline{\text { on }}_{\text {on the prism surface. The detailed }}$ process for the fabrication of the $\mathrm{SiO}_{2}$-based micro-fluidic channels is illustrated in Fig. 3 . Firstly, we formed a thin layer of $\mathrm{SiO}_{2}$ with a thickness of $1.5 \mu \mathrm{m}$ on the prism surface by wet oxidation. Then, we utilized the conventional photolithographic method to form grooves on $\underline{\text { the } \mathrm{SiO}_{2}} \underline{\text { layer which function as the micro-fluidic channels. Finally, a passivation layer of }}$ $\underline{\mathrm{SiO}}_{2}$ with $0.1 \mu \mathrm{m}$ thickwas formed on the bottom of the channels by dry oxidation. Dry oxidation has been carried out at an oxidation temperature of $1100^{\circ} \mathrm{C}$.

On the other hand, weopened uptwo holes in the quartz substrate that were used for the inlet and the outlet of sample solution. Finally, we utilized the conventional wafer bonding technique $[18,19]$ to bond the quartz substrate with the Si substrate to form micro-fluidic channelson the Si MIR-prism. Figures4(a) and 4(b) shows typical scanning electron microscope (SEM) images of the micro-fluidic channelsthat were formedon the Si MIR prism. Solutions of DNA in heavy water $\left(\underline{\mathrm{D}}_{2} \underline{\mathrm{O}}\right)$ were introduced intothe micro-fluidic channels due to capillary action. Figure 4(c) shows a photograph of the micro-fluidic channels we fabricated. Figure 5shows a photograph of the DNA solution moving through the channels. We can seethat the solution flowed uniformly in the channels; that is, the flow in each channel moved at almost the same speed. This indicatesthat the micro-fluidic channels we fabricated were rather uniform in size and shape.

\subsection{DNA samples}

30-mer oligonucleotides of adenine and cytosine were purchased and used without further purification. The single-stranded oligonucleotideswere solved in $\underline{\mathrm{D}}_{2} \underline{\mathrm{O}}$. The reason 
why we used므를 $\underline{O}$ instead of $\mathrm{H}_{2} \mathrm{O}$ as the solvent is that $\mathrm{H}_{2} \mathrm{O}$ has the strong scissoring peak around $1640 \mathrm{~cm}^{-1}$ where the bases of DNA exhibit specific vibration modes $(\mathrm{C}=\mathrm{O}, \mathrm{C}-\mathrm{N}$ stretching and also $\mathrm{NH}_{2}$ scissoring modes). On the other hand, $\underline{\mathrm{D}_{2}} \underline{\mathrm{O}}$ has no significant vibration modes at these wavelength regions; it has scissoring mode at $1230 \mathrm{~cm}^{-1}$. The

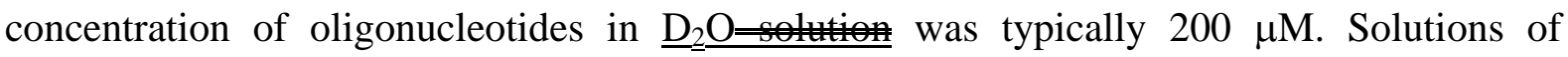
30-based oligonucleotides $\left(\mathrm{dA}_{30}, \mathrm{dC}_{30}\right)$ wereput into a small sample reservoir, while the micro-fluidic channels and a waste reservoir were filled with pure $\underline{\mathrm{D}}_{2} \underline{\mathrm{O}-\text { solution }}$. After that, aDC voltage was applied between the two ends of the micro-fluidic channels, that is, between the sample and waste reservoirs.Behavior of oligonucleotides moving through the micro-fluidic channels was monitored by MIR-IRAS to investigate the electrokinetic properties of DNA.

\subsection{MIR-IRAS measurement}

An infrared light beam from an interferometer (BOMEM MB-100) was focused at normal incidence onto one of the two bevels of the Si MIR-prism, and penetrated through the Si prism, internally reflecting about 60 times. The light that exited the Si prismthrough the other bevel on the opposite side was focused onto a liquid-nitrogen cooled mercury-cadmium-telluride (MCT) detector. The resolution of the interferometer was set at 4 $\mathrm{cm}^{-1}$. Since the refractive index of $\mathrm{Si}$, about 3.4, is larger than that of $\mathrm{SiO}_{2}$, about 1.5, internal reflection of infrared lights occurs at $\mathrm{SiO}_{2} / \mathrm{Si}$ interfaces. Strictly speaking, reflection at $\underline{\mathrm{SiO}_{2}}$ / 2 solution interface may occurs since the refractive index of $\mathrm{SiO}_{2} \underline{\text { is larger than that of }}$ $\underline{\text { water, approximately 1.3. However, if a } \mathrm{SiO}_{2}} \underline{\text { layer on } \mathrm{Si} \text { is much smaller than the penetration }}$ depth of the evanescent field, approximately $0.5 \mu \mathrm{m}$, we can regard the $\mathrm{SiO}_{2}$ layer as an absorbing medium layer. This is the case in the present experimental condition, since the

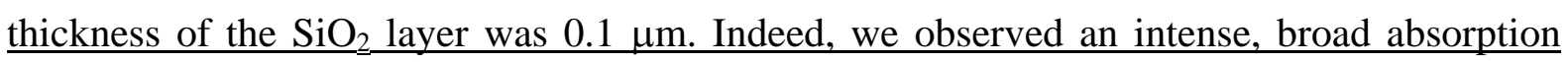


band around $1850 \mathrm{~cm}^{-1}$ for the MIR prism with the micro-fluidic channels. The broad absorption band may be attributed to the absorption of the $\mathrm{SiO}_{2}$ layers. However, this absorption was not too strong to obscure the absorption by the solution over the $\mathrm{SiO}_{2}$ layer. In addition, note that this absorption band was not overlapped with the absorption bands specific to DNA bases thatshould appear between 1500 and $1700 \mathrm{~cm}^{-1}$. When the oxide layer were thicker, exceeding the penetration depth, we would not able to monitor infrared absorption of the solution in microfluidic channels due to strong absorption of infrared lights

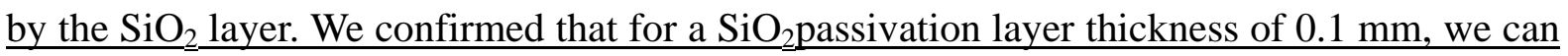
monitor infrared absorption of the solution over the $\mathrm{SiO}_{2}$ layer.Therefore, we can clearly distinguish the specific absorption bands of oligonucleotides moving in the micro-fluidic channels, as will be demonstrated in the next section.

\section{Results}

\subsection{IR spectra of DNA in the micro-fluidic channels}

Figure 6shows typical IRAS spectra collected for solutions of 30 -mer adenine $\left(\mathrm{dA}_{30}\right)$ and cytosine $\left(\mathrm{dC}_{30}\right)$ in $\underline{\mathrm{D}}_{2}$ O- that were introduced intothe micro-fluidic channels. For $\mathrm{dA}_{30}$, we can clearly identify two intense peaks at 1627 and $1560 \mathrm{~cm}^{-1}$. We have previously observed an intense peak at $1628 \mathrm{~cm}^{-1}$ on the spectrum collected for oligonucleotides of adenine floating in $\underline{D}_{2} \underline{O}$ [14-16]. We assigned the peak at $1627 \mathrm{~cm}^{-1}$ to the ring deformation mode of the base of adenine. The peak at $1560 \mathrm{~cm}^{-1}$ has not been observed in our previous works. Therefore, it could be due to impurities that had been contained in the sample solutions, most probably due to triethylammonium acetate (TEAA) which is widely used as a binding molecule in HPLC of DNA. For $\mathrm{dC}_{30}$, some peaks can be identified in the wavenumber region of $1500-1700 \mathrm{~cm}^{-1}$. These peaks can be attributed to vibration modes of the base of cytosine, except for the peak at $1560 \mathrm{~cm}^{-1}$. Thus, we confirmed that DNA molecules present 
in micro-channels can be detected with high sensitivity by the MIR-IRAS technique. Here, it should be pointed outthat the penetration depth of the infrared evanescent field is approximately $1 \mu \mathrm{m}$ for an infrared wavelength of $1000 \mathrm{~cm}^{-1}$, which is close to the height of our micro-fluidic channels, approximately $1.5 \mu \mathrm{m}$, as is shown in Fig. 4(a). This means that we can detect most of the DNA molecules moving through the channels. Furthermore, it should be noted that we can readily distinguish DNA molecules from undesired impurities through a quick inspection of infrared spectral profiles, as is shown in Fig. 6 . This is a great advantage of our method over other separation methods such as the conventional fluorescent techniques.

3.2. Real-time monitoring of DNA moving throughthe micro-fluidic channels

We have examined the drift of DNA solutions in the micro-fluidic channels; which force dominates in drifting DNA molecules in the channels: the force of electro-osmosis or that of electrophoresis. In me



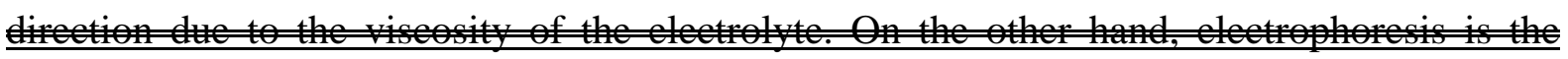

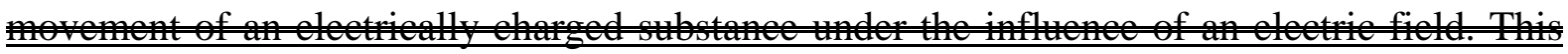
movent is due the Coulomb forcethats on the changed substance. In order to examine of $W e$ applied a potential of $50 \mathrm{~V}$ between two Pt electrodes located at the ends of each micro fluidic channel. Since oligonucleotides moved toward the cathode, we determined that electro-osmosis dominated in the present experimental condition. Therefore, we have in-situ collected MIR-IRAS spectra for 30-mer oligonucleotides, $\mathrm{dA}_{30}$ and $\mathrm{dC}_{30}$, drifting through the micro-fluidic channels due to the electro-osmotic force.

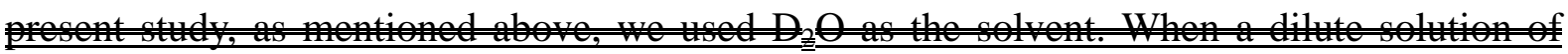
NaCl us as the solvent, the flow of DNA solution in the mico-fluidic chamels 
In order to monitorthe flow of DNA solutions in the micro-fluidic channels, we formed the so-called “plugs" of DNA solution by applying a DC voltage in a manner as depicted in Fig. 7. First, a solution containing DNA molecules was put into the sample reservoir and then was injected into the channels by electro-osmosis flow. After a short-term (approximately 1 min.) injection, the sample solution in the reservoir was replaced with $\underline{\mathrm{D}}_{2} \underline{\mathrm{O}}$ to produce sample plugs in the channels. After that, the sample plugs moved towards the waste reservoir through the micro-fluidic channels under the influence of an electric field.

Figure 8shows a series of IRAS spectra obtained for $\mathrm{dA}_{30}$ and $\mathrm{dC}_{30}$ molecules moving through the micro-fluidic channels. These spectra have been collected at intervals of 13 seconds. For $\mathrm{dA}_{30}$, the peak at $1627 \mathrm{~cm}^{-1}$, which is due to the ring vibration of the adenine base, initially showed up and then disappeared, as is shown in Fig. 8(a). This clearly indicates that sample plugs containing DNA ( $\left.\mathrm{dA}_{30}\right)$ crossedthepath of internally-reflecting infrared light. As can be seen in Fig. 8(b), similar results have been obtained for oligonucleotide of cytosine, $\mathrm{dC}_{30}$. The peak at $1650 \mathrm{~cm}^{-1}$ is due to the $\mathrm{C}=\mathrm{O}$ stretching vibration mode of the cytosine base. Thus, we can conclude thatmovement of DNA plugs in the micro-fluidic channels can be monitored using the MIR-IRAS technique.Furthermore, it should be noted that we can readily distinguish DNA molecules from undesired impurities through a quick inspection of infrared spectral profiles. As mentioned above, a broad peak around $1550 \mathrm{~cm}^{-1}$ is most probably due to TEAA which is widely used in the purification of DNA.Figure 8shows that this impurity can be distinguished from DNA molecules moving through the micro-channels. This is a great advantage of our method over other separation methods such 
as the conventional fluorescent techniques.

\section{Conclusions}

Infrared absorption spectroscopy (IRAS) in amultiple internal reflection (MIR) geometry was integratedwith silicon-based micro-fluidic channelsto allow detection and separation of DNA molecules in the micro-channels. The micro-fluidic channels were fabricated on a Si MIR-prism using the conventional silicon lithographic techniques. In the present method we can probeboth solution-phase and surface-bound biomolecular species through IR spectral profiles of those biomolecules, allowing us to detect and separate biomolecules without fluorescence labeling.The applicability of this approach wasdemonstrated with a representative test case: transport of single-stranded DNA molecules (oligonucleotides) in the micro-channels. The present method can be also applied to investigation on the behavior of other biomolecules in micro-fluidic channels.

\section{Acknowledgments}

This research has been carried out at the Laboratory for Nanoelectronics and Spintronics, Research Institute of Electrical Communication, Tohoku University. This work was partly supported by JST CREST. 


\section{References}

[1] D. J. Harrison, K. Fluri, K. Seiler, Z. Fan, C. S. Effenhauser, A. Manz, Micromachining a Miniaturized Capillary Electrophoresis-Based Chemical Analysis System on a Chip, Science 261 (1996) 895-897.

[2] J. W. Hong, V. Studer, G. Hang, W. F. Anderson, S. R. Quake, A nanoliter-scale nucleic acid processor with parallel architecture, Nature Biotech. 22 (2004) 435-439.

[3] H. Craighead, Future lab-on-a-chip technologies for interrogating individual molecules, Nature 442 (2006) 387-393.

[4] J. El-Ali, P. K. Sorger, K. F. Jensen, Cells on chips, Nature 442 (2006) 403-411.

[5] D. Schmalzing, A. Belenky, M. A. Novotny, L. Koutny, O. Salas-Solano, S. El-Difrawy, A. Adourian, P. Matsudaira, D. Ehrlich, Microchip electrophoresis: a method for high-speed SNP detection, Nucl. Acids Res. 28 (2000) e43.

[6] M. Ueda, Y. Kiba, H. Abe, A. Arai, H. Nakanishi, Y. Baba, Fast separation of oligonucleotide and triplet repeat DNA on a microfabricated capillary electrophoresis device and capillary electrophoresis, Electrophoresis, 21 (2000) 176-180.

[7] M. Ueda, T. Hayama, Y. Takamura, Y. Horiike, T. Dotera, Y. Baba, Electrophoresis of long deoxyribonucleic acid in curved channels: The effect of channel width on migration dynamics, J. Appl. Phys., 96 (2004) 2937-2944.

[8] M. C. Breadmore, Capillary and microchip electrophoresis: Challenging the common conceptions, Journal of Chromatography A, 1221 (2012) 42- 55.

[9] D. Proudnikov, A. Mirzabekov, Chemical methods of DNA and RNA fluorescent labeling, Nucl. Acids Res., 24 (1996) 4535-4532.

[10] A. L. Benvin, Y. Creeger, G. W. Fisher, B. Ballou, A. S. Waggoner, B. A. Armitage, Fluorescent DNA nanotags: supramolecular fluorescent labels based on intercalating dye arrays assembled on nanostructured DNA templates, J. Am. Chem. Soc., 129 
(2007) 2025-2034.

[11] M. Kölhed, P. Hinsmann, P. Svasek, J. Frank, B. Karlberg, B. Lendl, On-line fourier transform infrared detection in capillary electrophoresis, Anal. Chem. 74 (2002) 3843-3848.

[12] P. Svasek, E. Svasek, B. Lendl, M. Vellekoop, Fabrication of miniaturized fluidic devices using SU-8 based lithography and low temperature wafer bonding, Sensors and Actuators A 115 (2004) 591-599.

[13] M. Kölhed and B. Karlberg, Capillary electrophoretic separation of sugars in fruit juices using on-line mid infrared Fourier transform detection, Analyst, 130 (2005) 772-778.

[14] K. Miyamoto K. Ishibashi, K. Hiroi, Y. Kimura, H . Ishii, and M. Niwano, Label-free detection and classification of DNA by surface vibration spectroscopy in conjugation with electrophoresis, Appl. Phys. Lett. 86, 053902 (2005).

[15] K. Miyamoto, K. Ishibashi, R. Yamaguchi, Y. Kimura, H. Ishii, and M. Niwano, In situ observation of DNA hybridization and denaturation by surface infrared spectroscopy, J. Appl. Phys. 99, 094702 (2006).

[16] A. Hirano-Iwata, R. Yamaguchi,1 K. Miyamoto, Y. Kimura, and M. Niwano, In situ real-time monitoring of biomolecular interactions by using surface infrared spectroscopy, J. Appl. Phys. 105, 102039 (2009).

[17] N. J. Harrick, Internal Reflection Spectroscopy, Harrick Scientific Corp., Ossining, NY, 1967.

[18] H.Nakanishi, T. Nishimoto, R. Nakamura, A. Yotsumoto, T. Yoshida, S. Shoji, Studies on $\mathrm{SiO}_{2}-\mathrm{SiO}_{2}$ bonding with hydrofluoric acid. Room temperature and low stress bonding technique for MEMS, Sensors and Actuators, 79 (2000) 237-244.

[19] H.Nakanishi, T. Nishimoto, M. Kanai, T. Saitoh, R. Nakamura, T. Yoshida, S. Shoji, 
Condition optimization, reliability evaluation of $\mathrm{SiO}_{2}-\mathrm{SiO}_{2} \mathrm{HF}$ bonding and its application for UV detection micro flow cell, Sensors and Actuators, 83 (2000) 136-141. 


\section{Author Biographies:}

Tomoyuki Miyoshireceived the ME from Tohoku University in March, 2007 and received his $\mathrm{PhD}$ in electronics in 2015 from Tohoku University. He is working for Hitachi Central Institute, Hitachi Cooperation. His current research interest is fabrication of nanostructures and their applications to electronic devices.

Ken-ichi Ishibashireceived the ME from Tohoku University in March, 2005 and received his $\mathrm{PhD}$ in electronics in 2008from Tohoku University. He is working for Han-Ichi Cooperation. His current research interest is fabrication of nanostructures and their applications to electronic devices.

Ko-ichiro Miyamotoreceived his $\mathrm{PhD}$ in electronics in 2006from Tohoku University. He wasappointed research associate at Tohoku University in 2006. In 2013, he was appointed associateprofessor at the same university. His current research interest is fabrication of nanostructures and their applications to biosensingdevices.

Ayumi Hirano-Iwatareceived her PhD in chemistry in 1998from The University of Tokyo. She wasappointed research associate at Tohoku University in 2007. In 2009, she was appointed associateprofessor at the same university. Hercurrent research interest is fabrication of nanostructuresand lipid bilayers and their applications to electronic devices and biosensors.

YasuoKimurareceived his $\mathrm{PhD}$ in electronics in 1999 from The University of Tokyo. He wasappointed research associate at Tohoku University in 1999. In 2010, he was appointed associateprofessor at the same university. His current research interest is fabrication of nanostructures and their applications to electronic devices.

Michio Niwano received his $\mathrm{PhD}$ in physics in 1980 from Tohoku University. He wasappointed research associate at Miyagi University of Education in 1980. In 1987, he was appointed research associate at Tohoku University and appointed associateprofessor at the same university in 1989. In 1998, he started a research group atthe same university as a professor. His current research interest isnanofabrication forbioelectronics and nanoelectronics. 


\section{Figure captions:}

Fig.1: DNA detection system using micro-channel electrophoresis conjugated with MIR-IRAS.

Fig. 2: Procedure for the fabricationof micro-fluidic channels.

Fig. 3: Procedure for the formation of patterned $\mathrm{SiO}_{2}$ layers for micro-fluidic channels.

Fig. 4: (a) Cross sectional SEM image and (b) optical microscope image of the micro-channels fabricated on the Si prism surface. (c) Photograph of a DNA detection system with micro-channels.

Fig. 5: Photograph of the DNA solution moving through the micro-fluidic channels with 200 $\mu$ m width.

Fig. 6: Infrared absorption spectra of (a) $\mathrm{dA}_{30}$ and (b) $\mathrm{dC}_{30}$ stored in the micro-fluidic channels.

Fig. 7: Procedure of control of applied voltage for introduction of sample plugs.

Fig. 8: Time evolution of infrared absorption spectra of (a) $d A_{30}$ and (b) $d C_{30}$ moving through the micro-fluidic channels. 


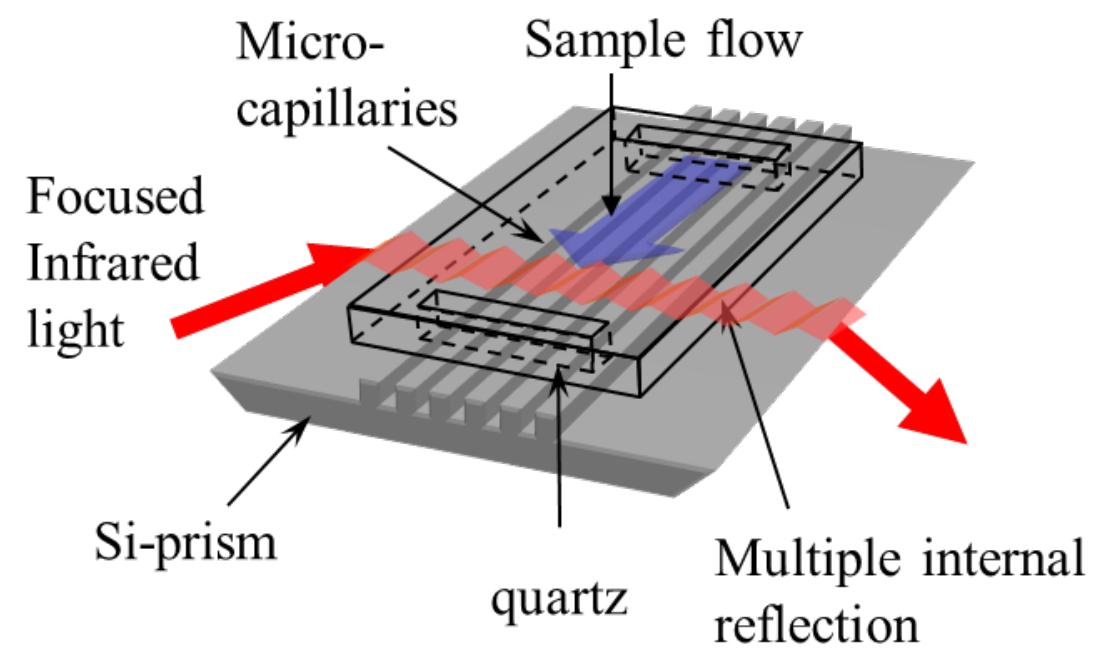

Cross-Sectional-View



Fig. 1 


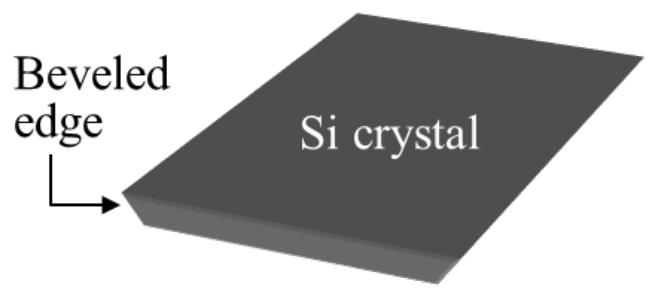

Preparation of a MIR Si prism



Quartz substrate





Formation of micro-channels on the prism surface

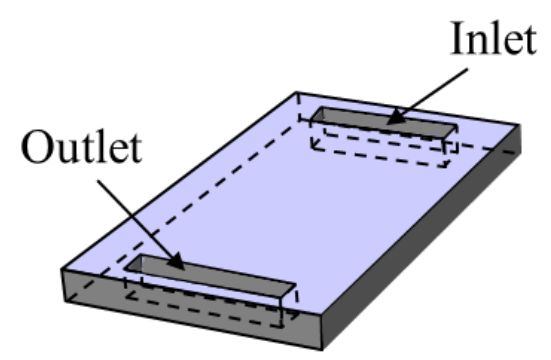

Puncture of holes for solution inlet and outlet

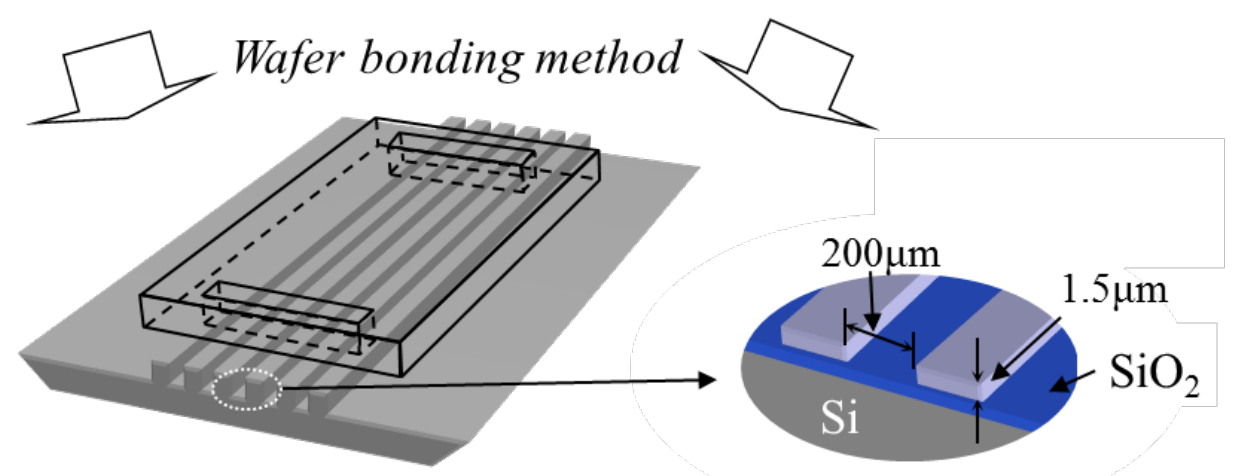

Fig. 2 
Beveled

edge

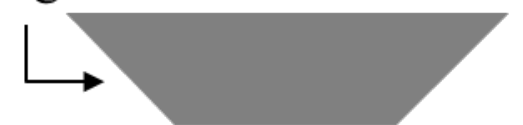

Preparation of a MIR Si prism Removal of patterned resist

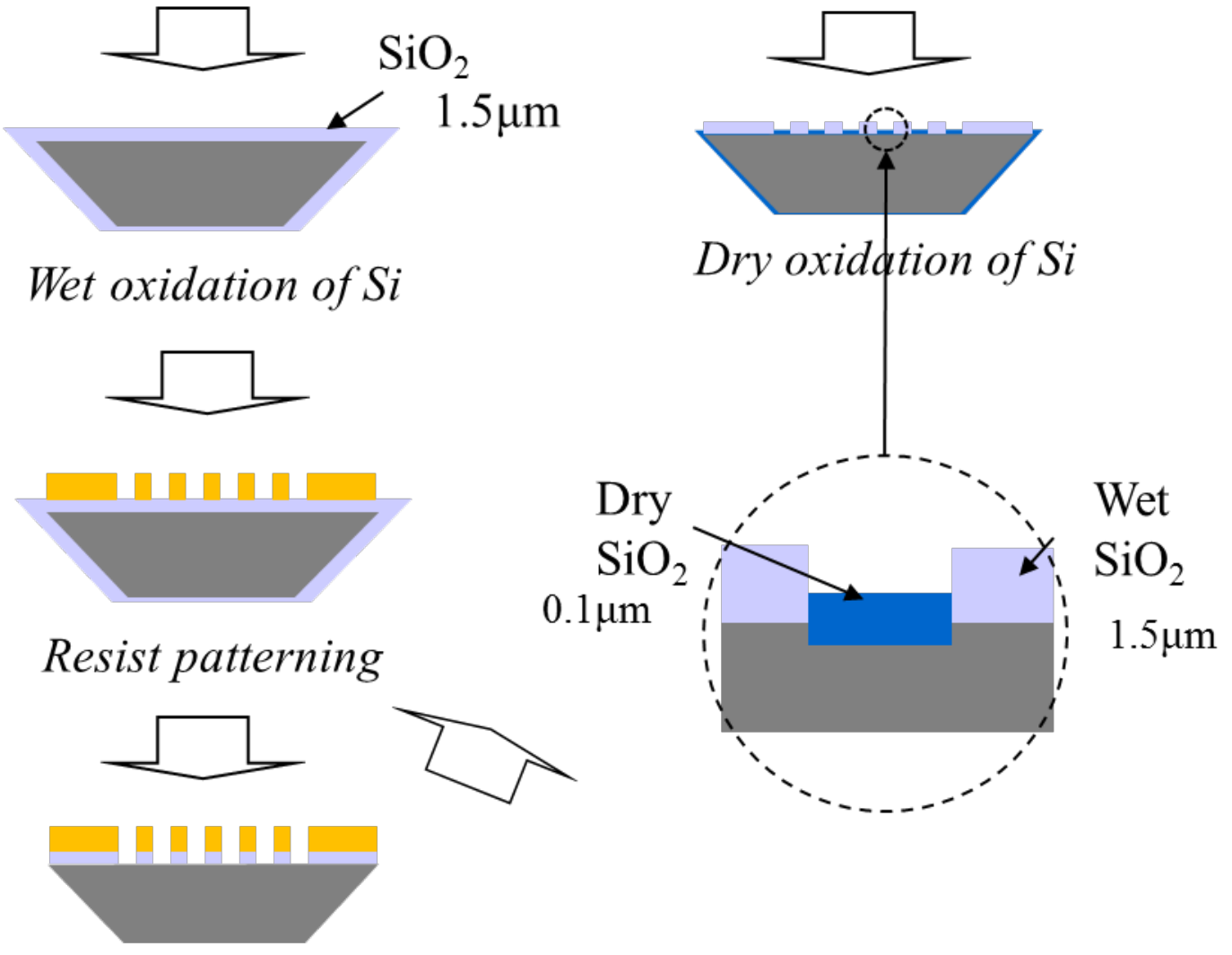

Wet etching of $\mathrm{SiO}_{2}$

Fig. 3 




Fig. 4 


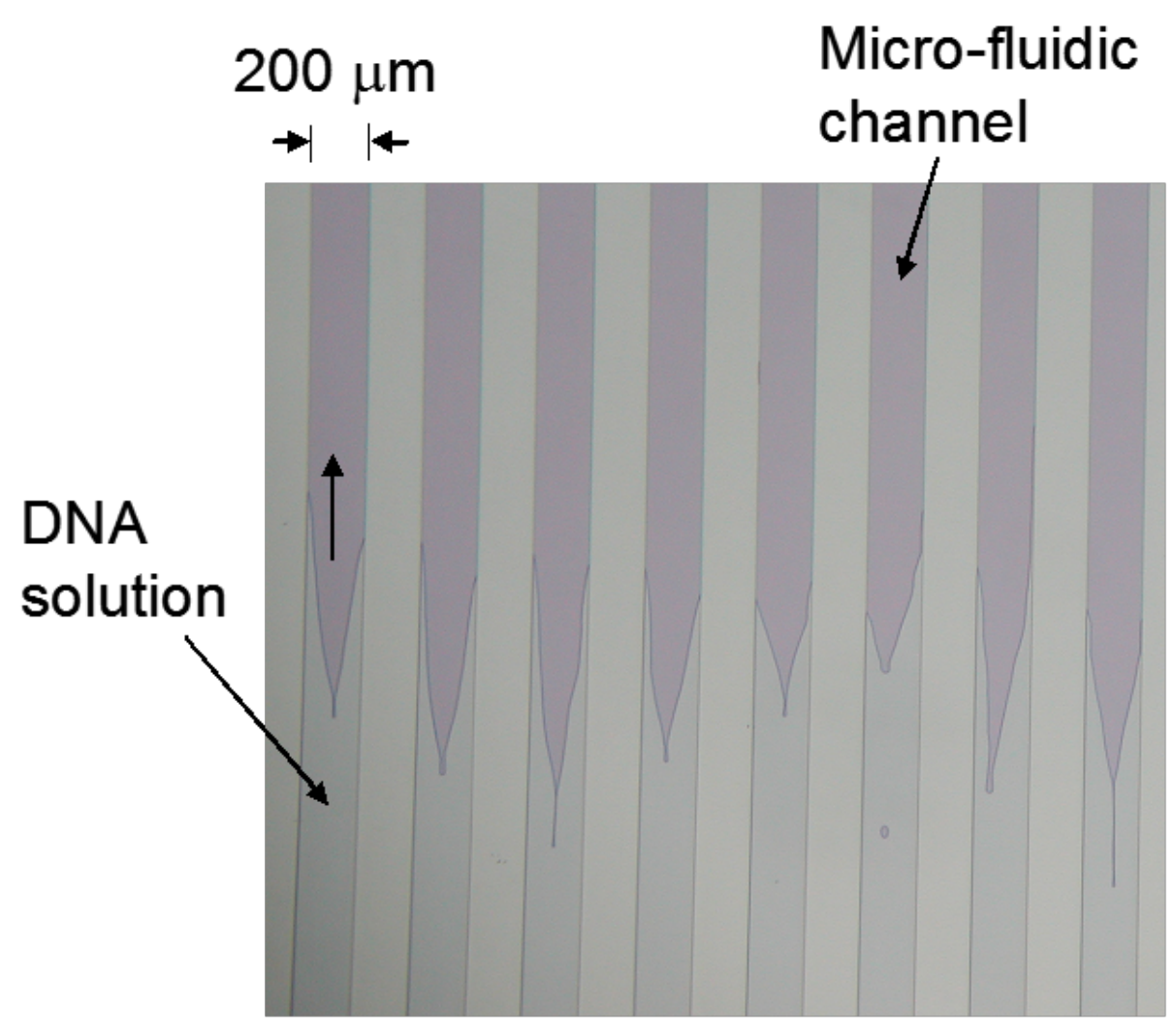

Fig. 5 


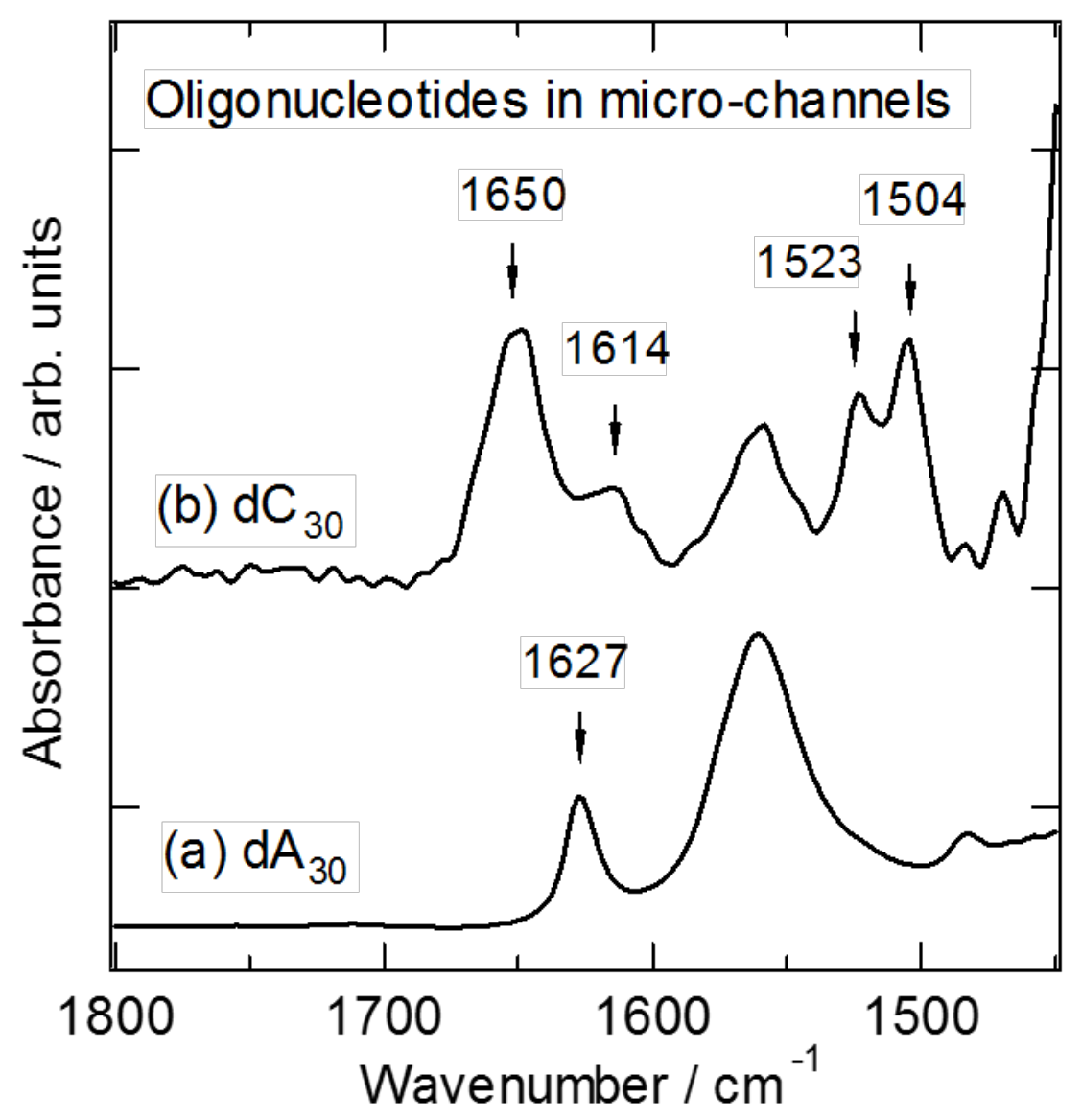

Fig. 6 


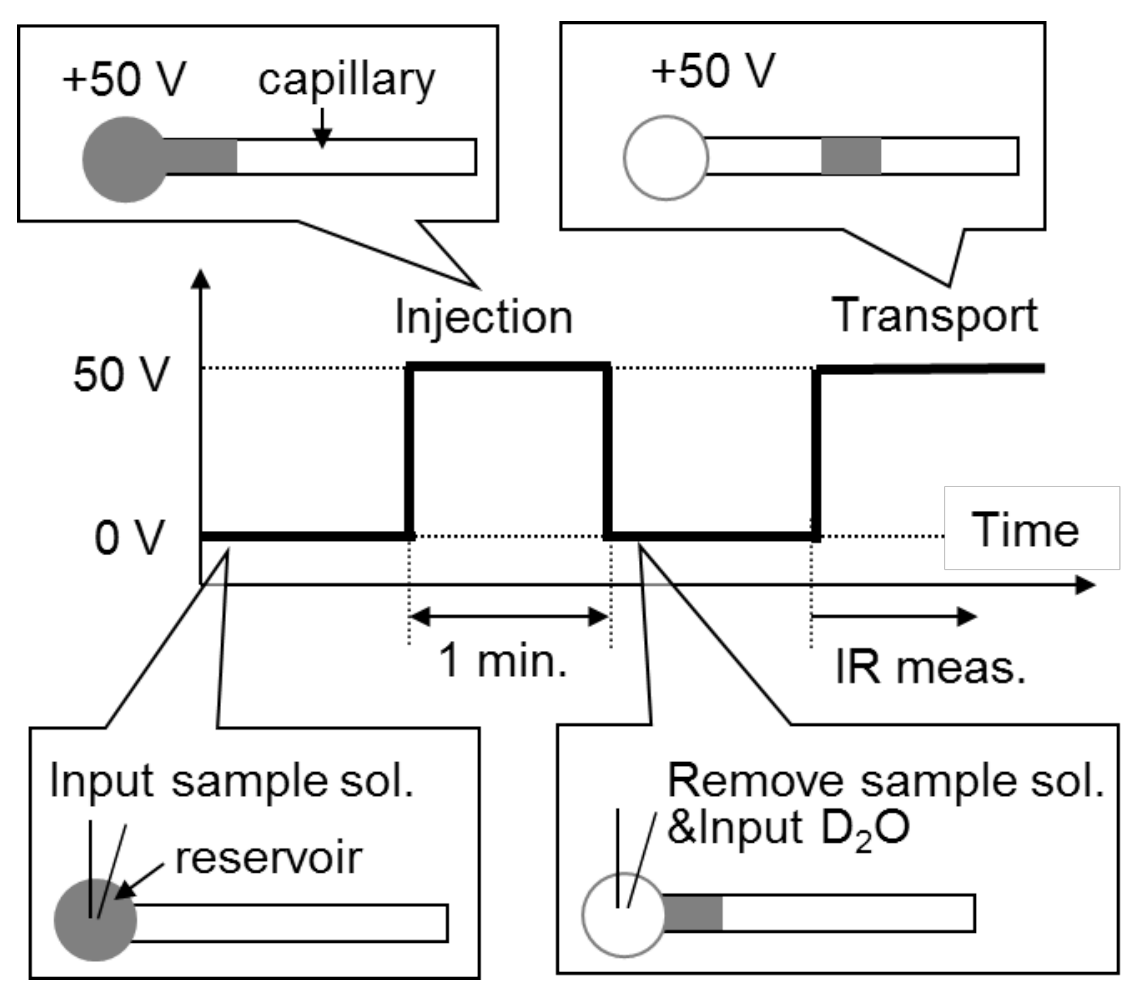

Fig. 7 



Fig. 8 